\title{
Vaccination Contribution to World Health: History, Current and Future
}

\author{
Hanaa A El Shafei ${ }^{1}$, Ebtsam M El Kady ${ }^{2 *}$ and Soad A Saleh ${ }^{2}$ \\ ${ }^{1}$ Microbial Chemistry Department, National Research Centre, Cairo, Egypt \\ ${ }^{2}$ Microbial Biotechnology Department, National Research Centre, Cairo, Egypt
}

*Corresponding author: Ebtsam Mokhtar El Kady, Microbial Biotechnology

Department, National Research Centre, Cairo, Egypt.

\begin{abstract}
Vaccination is the most effective way of avoiding widespread immunity from infectious diseases. For many parts of the world, vaccination is largely responsible for eradicating and preventing infectious diseases. Efforts to vaccinate have met with some controversy on scientific, legal, political, medical, and religious grounds, but no major religions condemn vaccination, and some find it a duty because of the potential for saving lives. Early success brought widespread recognition, and programs for mass vaccination dramatically decreased the incidence of many diseases in various geographic regions. Vaccination programs are seen as a major contributor to a 20th-century decline in infectious diseases. Over recent years, the national coverage of vaccination has declined, emphasizing the need for continuous monitoring and assessment of vaccination programmers.

Any vaccine is not completely risk-free, although most risks are very limited in adverse reactions to the vaccine. Therefore, we are working to produce a vaccine with a sufficient level of safety and positivity in the capacity for the required immunity. The different vaccine industry depends on the type of vaccine and the method of vaccination. Diphtheria is mainly regulated by vaccination, and by high immunization coverage ensures adequate herd immunity. The initiation of diphtheria outbreaks represents insufficient coverage of the vaccine. This epidemic was likely the result of the reintroduction by contaminated migrants passing through mining districts and poor vaccination levels of previously eradicated diseases. Work is still going on in developing, evaluating and discovering new forms of vaccines, including DNA vaccines, and recombinant vector vaccines which are simple and economical vaccines with good and long-term immune effects.
\end{abstract}

Keywords: Vaccines; Outbreaks; Diphtheria; Avidity

Abbreviations: DT-Diphtheria toxin; DTo-Diphtheria toxoids; ADP-Adenosine diphosphate; NAD-Adenine dinucleotide, DtxR-Diphtheria toxin repressor gene; (DT): diphtheria and tetanus; DTaP-Diphtheria tetanus and acellular pertussis; Td-Tetanus and diphtheria; Tdap-Tetanus, diphtheria, and acellular pertussis; (DAT)-Diphtheria antitoxin; DT(a)(w)P or TdaP-Acellular= a, wholecell=w; IPV-Polio virus; Abs-Antibodies; DTBS-Diphtheria toxin binding site; ELISA-Enzyme-Linked Immunosorbent Assay; MIA-Multiplex immunoassay; CV-Strong variability; HPV-Hepatitis virus

\section{Background}

Stimulating and activating the immune system against infectious diseases, by selecting vaccines in order to prepare the immune system, and this stimulation is called immune responses, which in turn provide acquired immunity to the disease against which the vaccine has been immunized, which usually consists of the microbe or its derivatives after weakening or destroying it. Vaccines may be prophylactic (to prevent or mitigate the effects of a potential infection by a natural or wild pathogen) or preventive (e.g. cancer vaccines under investigation) [1].
The inventions of Edward Jenner, which began with his popular 1796 [2] by using cowpox material to establish immunity to smallpox, rapidly spread the disease. At the beginning of the 19th century, Jenner's vaccine technique spread rapidly across the world, sponsored by preferred governments to a measure that could minimize the devastating effects of epidemics on their populations [3]. During the next 200 years, his process underwent scientific and technological changes and eventually led to the eradication of smallpox. 
The development of vaccines has increased significantly since the middle of the twentieth century, including the manufacture of virus vaccines in terms of development and innovations such as the polio vaccine and the triple vaccine (measles - German measles - parotid). This development was done by DNA and it is considered one of the most recent applications in the production and development of vaccines.

\section{Main text}

The different vaccine industry depends on the method of combating disease-causing germs and viruses by stimulating and stimulating the immune system. The response depends on the technology and approach of the vaccine, as there are four groups in the vaccine industry which are: polysaccharide, and conjugate vaccines. Subunit, recombinant, Live-attenuated vaccines, Inactivate vaccines.

Live-attenuated vaccines: In this type of vaccine, the microbe that causes the disease is used after it is weakened or destroyed, and this vaccine is given an effective and long-term immune response, and this immune protection is done by using one or two doses of this type of vaccine. Live vaccines are used to protect against: measles, mumps, rubella, rotavirus, smallpox, chickenpox, and yellow fever.

Inactivated vaccines: This type of vaccine does not provide good immunity like live vaccines, and this leads to the need for multiple doses of vaccination to obtain continuous immunity against the disease and among these diseases are hepatitis, influenza, polio and rabies.

Subunit, recombinant, polysaccharide, and conjugate vaccines: These types of vaccines use only part of the microbe, such as protein, sugars or capsid, as they target the main parts of the microbe which leads to a strong immune response. One of the negative aspects of this type is the need for multiple doses of the vaccine which use for immunized against Hepatitis B, Whooping cough, Shingles, Haemophilus influenzae type b disease, Human papillomavirus,, Pneumococcal disease, and Meningococcal disease.

Toxoid vaccines: Toxoid vaccines through converting the toxin into an toxoid in order to get rid of the toxicity of the vaccine, As the toxoid affects the immune part of the microbe and not the microbe itself which leads to the need for many doses (booster doses) of the vaccine in order to obtain permanent or continues protection. Toxoid vaccines are used to guard against: tetanus and diphtheria, diphtheria toxoid occur by cross linked the A-B fragments of diphtheria toxin.

Emerging outbreaks of toxigenic cutaneous diphtheria associated with conflict and failing healthcare systems: The crisis threatens to devastate the world's health-care system with significant morbidity and mortality implications. While emergency immunization programs are vital measures in the current situation to monitor and prevent outbreaks of infectious diseases in the long term. Diphtheria is also an issue with inadequate immunization coverage in a number of low-income countries. Several outbreaks in Sub-Saharan Africa were recorded e.g. Nigeria and Madagascar since 2000 [4]. Recently in South-East Asia the diphtheria outbreaks such as the 2012-2013 People's Democratic Republic of Lao [5] and 2015 and 2016 in India [6]. In 2017 there was a diphtheria outbreak among the Pemon ethnic group Amerindians in Wonken, Venezuela [7]. Last but not least, the recent ongoing diphtheria outbreak in Yemen2019 exposed the increasing number of cases of diphtheria and deaths as well as the recent shortages of antitoxin diphtheria and cases leading to immunization coverage failure [8]. WHO European region reported the shortages of diphtheria antitoxin, the Strategic Advisory Group of Experts (SAGE) on immunization called for a review of the evidence available and the need to reexamine the current recommendation. Now let's get to know about this illness, its causes, symptoms and ways of defending us against it.

\section{Diphtheria}

It is a serious bacterial infection caused by the bacteria Corynebacterium diphtheria [Figure1]. Diphtheria causes the back of the throat to have thick covering. This can lead to breathing difficulties, heart failure, paralysis and even death. The average diphtheria case-fatality is 5-10 percent. Corynebacterium diphtheria has 4 subspecies (gravis, mitis, belfanti and intermedius). One of the most common dangers of diphtheria is respiratory infection, and it also affects the pharynx and tonsils. In severe cases, pseudo-obstructive membranes form in the respiratory system, and complications of diphtheria are myocarditis and neuritis. Diphtheria may occur in a cutaneous form, resulting in indolent skin infection.

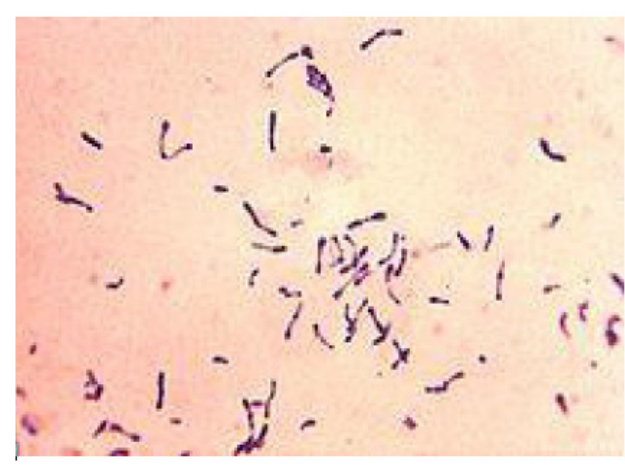

Figure 1: Corynebacterium diphtheriae the pathogenic bacterium that causes diphtheria.

Diphtheria toxin: It is an exotoxin which is secreted by the pathogenic causing diphtheria Corynebacterium diphtheriae. A prophage (a virus that has itself inserted into the host bacterium's genome) encodes the toxin gene. It is responsible for the Diphtheria symptoms. By 1888 the poison was isolated, in the year 1923 a vaccine was made. Diphtheria toxin (DT) contains three structural domains, each with a distinct biological function involved in cell 
poisoning: cell-surface binding and internalization into endosomes, crossing of the endosome membrane into the cytosol, and preventing the synthesis of cell proteins.

Pathogenicity of Diphtheria Toxin: Diphtheria toxin is a protein that contains 535 residual amino acids. It is synthesized as a single polypeptide, but it is proteolyzed in its active form to two polypeptide chains connected by a disulfide bond. The fragment C-terminal B (345 residues) contains the transmembrane and receptor binding domains, and the fragment N-terminal A (190 residues) contains the catalytic domain [Figure 2]. On the cell surface, diphtheria toxin binds to the precursor of the heparinbinding epidermal growth factor, and the complex of toxin-receptors undergoes endocytosis mediated by receptors. Next, the A fragment is translocated into the cytosol across the endocytic membrane. The catalytic domain, once in the cytosol, catalyzes the transition of adenosine diphosphate (ADP) ribose from nicotinamide adenine dinucleotide (NAD) to elongation factor 2 , inhibits protein synthesis and results in cell death [9].

Figure 2: Tri-dimensional arrangement of the diphtheria toxin functional domains. In the CRM197 version, highlighted amino acid glycine at position 52 is substituted with glutamine.
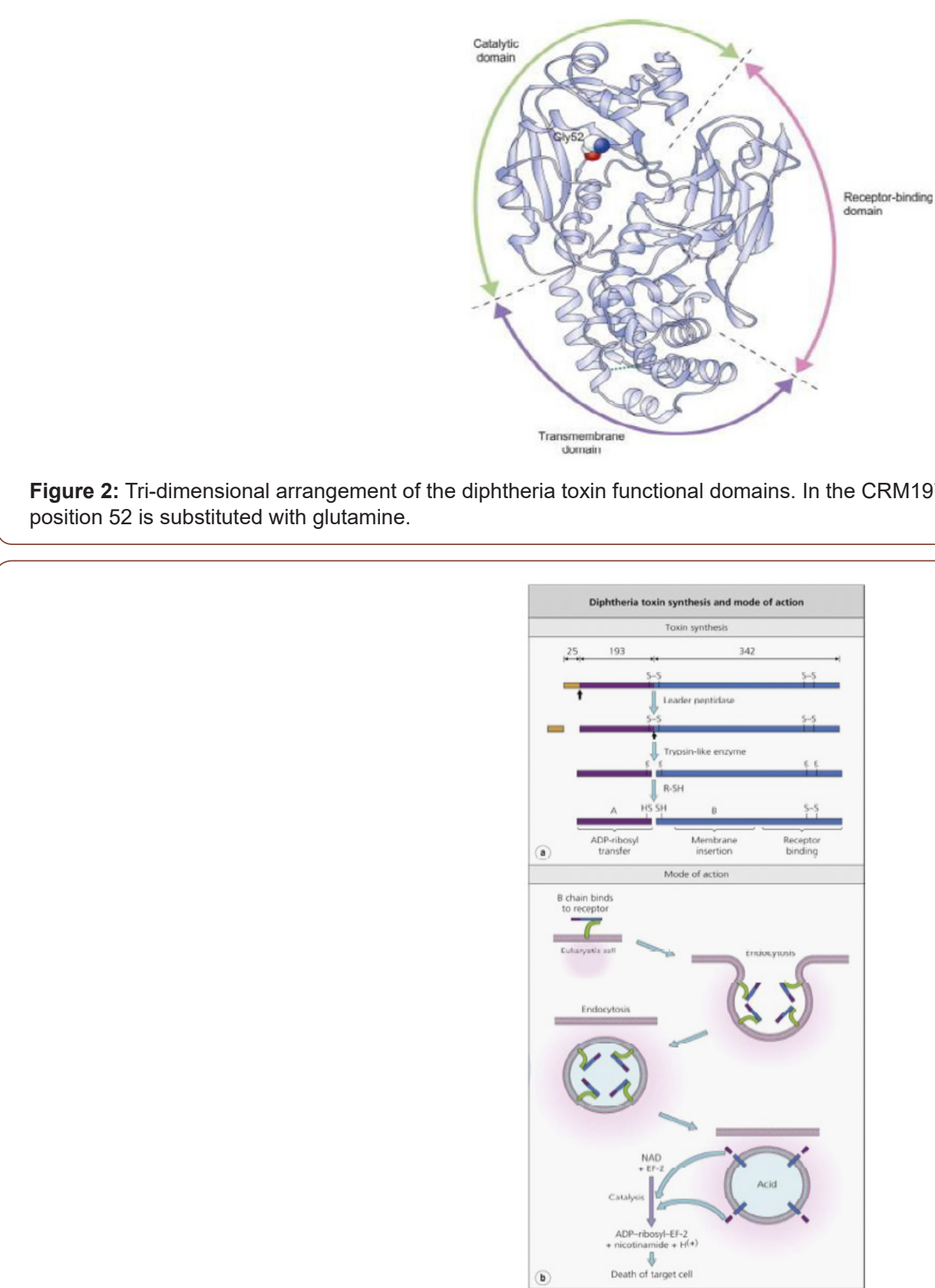

Figure 3: Synthesis of diphtheria toxins, and mode of action. (A) The 25-residue leader sequence is cleaved off by the bacterial leader peptidase; the' trypsin-like enzyme' releases the A and B subunits from the precursor protein. Once in the cytoplasm of a targeted eukaryotic cell, the A chain responsible for the transfer of ADP-ribosyl is disconnected from the B chain, responsible for the binding of the receptors and membrane insertion. (b) The chain B binds the eukaryotic cell to a particular receptor. After endocytosis, endosome acidification causes the $\mathrm{B}$ chain to be incorporated into the endosomal membrane and subunit A to be translocated into the cytosol, where it catalyzes the EF-2 ribosylation of the ADP. Protein synthesis is therefore prevented and the targeted cell dies. 
Nature and Pathogenicity of Microorganisms: Diphtheria toxin is a part of the so-called bifunctional toxins A-B. Fragmentart $A$ is the enzyme activity responsible for halting protein synthesis in the target cell, while Fragment B is related to the cell receptor as it prevents the transfer of Fragment $\mathrm{A}$ into the cytosol Portion $B$ accounts for the cell and species specificity of the A-B toxins. Fragment B of diphtheria toxin deals with a heparin-binding precursor of epidermal growth factor, which is an essential hormone for cell growth and differentiation. Uptake of diphtheria toxin is done through endocytosis mediated by the receptor Endocytic vesicle acidification induces a conformation of the enclosed holotoxin, allowing Fragment A subunit of the diphtheria toxin to traverse the membrane and reach its cytoplasmic target. The A subunit of diphtheria toxin catalyzes the elongation factor- 2 (EF-2) ribosylation of ADP and inactivates it [Figure 3].

The tox gene is encoded by a phage and is controlled by the DtxR (diphtheria toxin repressor gene )suppressor protein, which forms an iron complex, DtxR-Fe that binds DNA and restrains the expression of tox [Figure 4].Therefore, diphtheria toxin is only synthesized under low iron conditions, indicating that iron release from target cells can be stimulated $[10,11]$.

Diphtheria Vaccination: Four forms of vaccines used today to protect against diphtheria and other diseases as well: Vaccines for diphtheria and tetanus (DT), diphtheria, tetanus, and acellular pertussis (DTaP), tetanus and diphtheria (Td), tetanus, diphtheria, and acellular pertussis (Tdap). Babies and kids under the age of 7 receive DTaP or DT while older kids and adults receive Tdap and Td.

Passive and active immunization: Passive immunization of equine origin through diphtheria antitoxin (DAT) is highly effective in the treatment of diphtheria though it is not a substitute for active immunization using diphtheria toxoid. Nonetheless, antitoxin is an effective diphtheria medication, and can reduce morbidity as well as mortality. Should be administered diphtheria antitoxin (DAT) as soon as possible after the initiation of the disease, once the toxin has reached the host cells the antitoxin is unaffected. You will administer the entire therapeutic dose at one time. The recommended amount of antitoxin ranges from 20,000 to120,000 units with larger amounts recommended for individuals with severe local lesions and longer periods since the onset. New approaches include developing monoclonal antibodies to diphtheria toxin or developing recombinant modified toxin receptor molecules to bind toxin to diphtheria. To date, however, no monoclonal diphtheria toxin antibody has been approved for clinical use, so treatment is still based on DAT. The toxoid to diphtheria is used for successful immunization. Diphtheria vaccines are based on the toxoid diphtheria, a modified bacterial toxin that causes defensive antitoxin. immunization with DT by combination the diphtheria toxoid with tetanus toxoid as DT (for use $<7$ years of age) or Td (for use $<7$ years of age) or as DT(a)(w) P or TdaP with tetanus and pertussis vaccine (acellular $=\mathrm{a}$, wholecell $=\mathrm{w}$ ). Diphtheria toxoid associated with other vaccine antigens such as polio (IPV), hepatitis B, and type b Haemophilus influenzae.

\section{The efficacy of diphtheria antitoxin antisera}

The antigen binding with the corresponding Abs determines the basis for evaluating vaccines, therapeutic antisera, and human and population immune functions. Composition of the antigen produced, aside from assay types, Abs complex interaction is mainly determined by reactant heterogeneity, interaction specificity, and reactant concentration. These interactions were widely used in many assays, in which one of the reactants was calculated by the arbitrary set-end stage. A variety of methods are commonly used to titrate diphtheria antitoxins, including both in vivo and in vitro assays. While in vivo study has the advantage of testing the Abs, which essentially neutralizes the toxin, it is relatively costly and repeatable. Several complementary quantitation assays have been developed for DT and antitoxins [12,13]. The total Abs content and the Abs avidity have not been precisely calculated for any of those measures. Although the measured traits could be very distinct in different assays, the determined titers were found to be satisfactory in various applications. The heterogeneity of contributing factors was essentially largely overlooked. Abs 'avidity was recognized as an effective vector in restraining the defense against various diseases [14].

Avidity as a criterion for the efficacy of the vaccine against diphtheria: The term avidity refers to how closely it binds an antibody to their antigen. Affinity refers to the strength of the bond between an antibody and its antigen. However, several isotypes of antibodies are multivalent, and bind to several antigens. The strength of this overall partnership is the covetousness of avidity. Antibodies (Abs) avidity to diphtheria toxin (DT), toxoids (DTo) and the diphtheria toxin binding site (DTBS) were investigated in sera from guinea broad vaccinated with specific DTo during 2004. Measuring avidity by the thiocyanate method showed that when maturing, Abs maturation trends to the corresponding DTo were not quite different. In the DTBS affinity variations of up to 20 folds were observed as calculated by the tissue culture technique and expressed as equilibrium constant $(\mathrm{K})$. Abs 'avidity to either the corresponding DTo or the DT could not be correlated with the vaccine's effectiveness, while the vaccine's efficacy could be measured by its association with the DTBS. This can be represented in any procedure in terms of measuring precision. The thiocyanate procedure measures the average avidity to complex antigens with multiple epitopes while the tissue culture procedure allows the DTBS affinity of Abs to be determined. It is concluded that the priority in testing new vaccines should involve measuring the avidity of Abs to the known protective epitome [15].

Defense against different pathogens depends mainly on antibodies, where each antibody is specific to a certain antigen to reach maximum effect and this is the major part of humoral immunity. A research to determine the relevance and reliability 
of the non-functional Enzyme-Linked Immunosorbent Assay (ELISA) for potency testing of diphtheria toxoid-containing combination vaccines was initiated in January 2000 [16]. According to conventional antibody standard, a strong enzyme-linked immunosorbent assay (ELISA) was proven to identify the quantity of anti-diphtheria antibodies in human serum, researches showed very accurate results where recovery have reached up to 97.06 $\%$. The ELISA test mentioned for the quantitation of diphtheria antitoxin is a valuable tool for evaluating immunological defense against diphtheria and could be particularly useful for population studies, since it is economical and practical for large-scale routine purposes.

A quick hexavalent bead-based method was developed by [17] to improve preclinical assessment of serological immune responses to the individual components of DTP combination vaccines diphtheria, tetanus, and pertussis (DTP). The sensitivities of the mouse DTP avidity multiplex immunoassay (MIA) per antigen were comparable to those of the six individual in-house avidity ELISAs, and strong correlations of the IgG concentrations obtained for all antigens tested by both methods were shown. The normal and active mouse DTP MIAs were reproducible for all antigens, with strong variability (CV) intra- and inter-assay coefficients. Ultimately, a retrospective study of the production and avidity maturation of differentIgG antibodies in mice demonstrated the utility of the assay. They conclude that the hexaplex mouse DTP MIA is a reliable, responsive and high-throughput alternative for ELISA in preclinical vaccine studies to investigate the quantity and quality of serological responses to DTP antigens [18]. Estimate avidity by a Modified ELISAs by using chaotropic agents and calculating the degree to which they interfere with the interaction between the antigen and the antibody. The idea behind the test is the greater an interaction's avidity the less sensitive it is to the effects of the chaotropic agent. The test was highly reproducible and identified a wide variety of avidities for antibodies. Consequently, a GuHCl-modified ELISA is an appropriate approach which can be used within a clinical trial setting to evaluate HPV-specific antibody avidity indices.

\section{Conclusion}

Diphtheria is mainly regulated by vaccination, and by high immunization coverage ensures adequate herd immunity. The initiation of diphtheria outbreaks represents insufficient coverage of the vaccine. This epidemic was likely the result of the reintroduction by contaminated migrants passing through mining districts and poor vaccination levels of previously eradicated diseases [19]. In addition to several outbreaks, thousands of cases of diphtheria are still recorded annually from many countries in Asia and Africa. Changes in diphtheria epidemiology have been identified across the globe. Toxigenic Corynebacterium is prevalent. Highlights the need for effective clinical and epidemiological investigations with a view to rapid diagnosis and care of sick persons and public health.
Additional studies for new assays and limits are needed to increase the current level of vaccine potency. These attempts to improve assays are expected to stimulate the production of the diphtheria vaccine and lead to self- of the vaccine.

\section{Declarations}

Ethics approval and consent to participate Not applicable.

\section{Consent for publication}

Not applicable.

\section{Availability of data and materials}

All information and data included in this study are included in this manuscript.

\section{Competing interest}

The authors declare that they have no competing interests.

\section{Funding}

Not applicable.

\section{Authors' contributions}

Prof HAE, EME and SAS shared equally in writing and revising the manuscript and they approved the final version.

\section{Acknowledgement}

Thanks and appreciations for members of Microbial Chemistry and Microbial Biotechnology Departments for their support.

\section{Conflict of Interest}

No conflict of interest.

\section{References}

1. Melief CJ, van Hall T, Arens R, Ossendorp F, van der Burg SH (2015) "Therapeutic cancer vaccines". J Clin Invest 125(9): 3401-12.

2. Alexandra MS, Howard M (2005) The History of Vaccines and Immunization: Familiar Patterns, New Challenges. Health Affairs 24 (3).

3. Lombard PM, Pastoret, Moulin AM (2007) A brief history of vaccines and vaccination. Rev sci tech Off int Epiz 26 (1): 29-48.

4. Clarke K (2017) Reveiw of the epidemiological of Diphtheria -20002016. US centers for diseases control and prevention.

5. Sein C, Tiwari T, Macneil A, Wannemuehler K, Soulaphy C, et al. (2016) Diphtheria outbreak in Lao People's Democratic Republic, 2012-2013. Vaccine 34(36): 4321-4326.

6. Das PP, Patgiri SJ, Saikia L, Paul D (2016) Recent Outbreaks of Diphtheria in Dibrugarh District, Assam, India. J Clin Diagn Res 10(7): DR01-DR03.

7. Lodeiro Colatosti A, Reischl U, Holzmann T, Hernández Pereira CE, Rísquez A, et al. (2018) Diphtheria Outbreak in Amerindian Communities, Wonken, Venezuela, 2016-2017. Emerg Infect Dis 24(7): $1340-1344$.

8. Dureab F (2019) Diphtheria outbreak in Yemen: the impact of conflict on a fragile health system. Confl Health 13: 19.

9. Kabanova A, Rappuoli R (2011) Tropical Infectious Diseases ( $3^{\text {rd }}$ edn).

10. Daniel G, Julien B (2015) The Comprehensive Sourcebook of Bacterial Protein Toxins $\left(4^{\text {th }}\right.$ edn). 
11. Fierer J, Pechère J (2017) Infectious Diseases ( $4^{\text {th }}$ edn).

12. Wahby AF, El Ridi R, Abd El Sami M, El Kady EM (1991a) Cytotoxicity and Equilibria in mixtures containing diphtheria toxin.I-Correlation of the toxin induced cell growth inhibition and in vitro titers to toxin concentrarion. J Egypt Ger Soc Zool 6(A): 211-232.

13. Wahby AF, El Ridi R, Abd El Sami M, El Kady EM (1991b) Cytotoxicity and Equilibria in mixtures containing diphtheria toxin. II-Relative neutralization potency of antibioties to the toxin binding site. J Egypt Ger Soc Zool 6(A): 233-250.

14. Gupta R, Emili A, Pan G, Xiao H, Shales M, et al. (1996) Characterization of the interaction between the acidic activation domain of VP16 and RNA polymerase II initiation factor TFIIB. Nucleic Acid Res 24 (12): 23242330.

15. El Kady EM (2004) Avidity as a parameter for diphtheria vaccine efficacy. Egypt J Immunol 11(1): 47-54
16. Martínez Rodríguez JC, Azze RO, Perón AC, Fajardo EM, Figueredo EA, et al. (2000) Validation of an ELISA for the Quantitation of Diphtheria Antitoxin in Human Serum. Biotecnología Aplicada 17: 183-186.

17. Stenger RM, Smits M, KuipersB, Kessen SFM, Boog CJP, et al. (2011) Fast, Antigen-Saving Multiplex Immunoassay To Determine Levels and Avidity of Mouse Serum Antibodies to Pertussis, Diphtheria, and Tetanus Antigens. Clin Vaccine Immunol 18(4): 595-603.

18. Dauner JG, Pan Y, Hildesheim A, Kemp TJ, PorrasC, et al. (2012) Development and application of a GuHCl-modified ELISA to measure the avidity of anti-HPV L1 VLP antibodies in vaccinated individuals. Mol Cell Probes 26(2): 73-80.

19. Mac Gregor RR (2010) Corynebacterium diphtheriae. In: Mandell, Douglas, and Bennett's Principles and practice of infectious diseases, 7 th ed. Philadelphia: Churchill Livingstone Elsevier 2687-2693. 